\title{
Vitiligo y terapia con inmunoglobulina intravenosa: a propósito de un paciente con síndrome de Sjögren
}

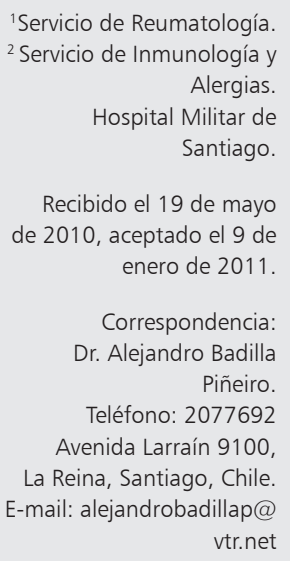

\author{
ALEJANDRO BADILLA P. ${ }^{1}$, CAROLINA DÍAZ G. ${ }^{2}$
}

\section{Remission of vitiligo during treatment with intravenous immunoglobulin. Report of one case}

Vitiligo is associated with other autoimmune diseases. We report a 52-year-old male with a Sjögren syndrome that was treated with monthly pulses of intravenous immunoglobulin for a chronic inflammatory demyelinating polyradiculoneuropathy. The neurological disorder responded adequately to the treatment and the patient also noted a marked remission of his vitiligo with almost compete re-pigmentation of the scalp and face and partial re-pigmentation of other areas.

(Rev Med Chile 2011; 139: 484-488).

Key words: Immunoglobulins; Polyradiculoneuropathy; Vitiligo.
$\mathrm{E}$ l vitiligo es un trastorno de la pigmentación cutánea, caracterizado por la presencia de áreas de piel despigmentada, secundario a la pérdida crónica y progresiva de los melanocitos epidérmicos. A nivel mundial tendría una prevalencia entre $0,5-2 \%$ según las diferentes series, aunque en estudios recientes se ha sugerido que esta cifra podría estar sobrestimada ${ }^{1}$.

Su etiología es aún incierta, sin embargo, ha sido establecido que en pacientes genéticamente susceptibles, factores como la autoinmunidad, las infecciones, el estrés, anormalidades neurológicas, disfunciones en el receptor de la melatonina, de la migración del melanocito y de origen tóxico, entre otros, podrían tener un rol patogénico².

En relación al proceso autoinmune, existiría participación directa del sistema inmunológico, tanto de la rama humoral como celular. La primera se caracteriza por presencia de anticuerpos contra múltiples antígenos expresados en los melanocitos y la segunda por el reclutamiento de linfocitos $\mathrm{T}$ (LT) CD8+ citotóxicos y macrófagos. Posiblemente, la destrucción inicial de los melanocitos sería consecuencia de la acumulación de metabolitos tóxicos y posterior amplificación autoinmune del daño sobre éstos.
Clínicamente puede manifestarse en forma segmentaria o difusa, asociándose esta última variedad a diversas enfermedades autoinmunes, entre las que destacan la tiroiditis de Hashimoto, el lupus eritematoso sistémico y la anemia perniciosa. Estas etiologías autoinmunes representan $30 \%$ del total de pacientes con la forma difusa ${ }^{2}$.

Desde el punto de vista psicosocial, el vitiligo puede tener un alto impacto en la autoestima y calidad de vida del paciente afectado, incluso mayor al asociado con la psoriasis.

Actualmente, disponemos de diversas aproximaciones terapéuticas para esta patología, las que dependen principalmente de la extensión del compromiso cutáneo y del tipo de piel, sin embargo, no existe hasta el momento un tratamiento completamente efectivo ${ }^{3}$.

La terapia con inmunoglobulina intravenosa (IGIV) posee un complejo mecanismo de acción. Someramente podemos resumir sus funciones en: modulación de la expresión y función de los receptores $\mathrm{Fc}$, interferencia con la activación del complemento y la red de citoquinas, provisión de anticuerpos anti-idiotipo, modulación de las células dendríticas e interferencia con la activación y función de los linfocitos B y T, siendo esta última, 
Inmunoglobulina intravenosa como terapia de vitiligo - A. Badilla et al

al parecer, su principal mecanismo de acción ${ }^{4}$. Los receptores Fc corresponden a moléculas presentes en la membrana celular de diversas células, que permiten la unión de la fracción Fc de las inmunoglobulinas, mediando diferentes efectos fisiológicos, como la opsonización, la lisis celular, entre otros.

Además de su uso como terapia de reemplazo en las inmunodeficiencias, la IGIV se ha utilizado, en dosis mayores $(1-2 \mathrm{~g} / \mathrm{kg})$ y con fines inmunomoduladores, en diversas enfermedades autoinmunes con evidencia en estudios clínicos controlados (Tabla 1$)^{4}$. Existe en la literatura una extensa lista de otros ejemplos de casos y series clínicas en que ha sido utilizada exitosamente ${ }^{5}$. En todas estas situaciones su indicación no es de rutina sino que se reserva para los pacientes de difícil manejo, que no han respondido a las terapias clásicas.

A continuación se presenta un caso clínico en el cual, sin tener como objetivo primario el tratamiento del vitiligo, en forma coincidente con el uso de IGIV, se observó una sorprendente y rápida repigmentación de amplias zonas comprometidas.

\section{Caso clínico}

Paciente de sexo masculino, 52 años, con antecedentes de hipotiroidismo secundario a una tiroiditis crónica, síndrome de Sjögren, insulinoresistencia y vitiligo generalizado de aproximadamente 34 años de evolución, manejado en dermatología sin buenos resultados.

Asociado al síndrome de Sjögren, el paciente presentó un cuadro neurológico interpretado como una poliradiculoneuropatía desmielinizante inflamatoria crónica (CIDP). Inició tratamiento con prednisona $1 \mathrm{mg} / \mathrm{kg} / \mathrm{d}$ en diciembre de 2007, el que mantuvo con disminución progresiva de la dosis hasta junio de 2008. No evolucionó favorablemente con este tratamiento, desarrollando un síndrome de Cushing secundario. Por lo anterior, se presentó al equipo de reumatología y, considerando el fracaso a coticoesteroides, se decidió iniciar pulsos mensuales de IGIV. Durante los primeros dos meses se utilizan dosis inmunomoduladoras $(2 \mathrm{~g} / \mathrm{kg})$, las que luego se disminuyeron paulatinamente hasta llegar a $0,5 \mathrm{~g} / \mathrm{kg}$, debiendo ser incrementada por reactivación de la neuropatía a $1 \mathrm{~g} / \mathrm{kg}$.
Tabla 1. Indicaciones de IGIV con evidencia en estudios clínicos controlados

\begin{tabular}{l}
\hline Púrpura trombopénico idiopático \\
Síndrome de Guillain-Barré \\
Polineuropatía desmielinizante inflamatoria crónica \\
(CIDP) \\
Neuropatía motora multifocal \\
Miastenia gravis \\
Enfermedad de Kawasaki \\
Dermatomiositis \\
Enfermedades cutáneas ampollares autoinmunes \\
Enfermedad de injerto contra huésped \\
\hline
\end{tabular}

Desde la primera infusión presentó una franca recuperación del cuadro neurológico, la que se mantiene hasta ahora. Después del cuarto mes de tratamiento, el paciente refiere en forma espontánea una marcada disminución del vitiligo con repigmentación casi total de cara, cuero cabelludo, pabellones auriculares, región cervical y en alrededor de 50\% en manos, pies y zona genital. Lo anterior se pudo objetivar gracias a varias fotografías que el paciente se había tomado, por iniciativa propia, antes de iniciar el tratamiento (Figuras 1A y 1B). Hasta el momento ha completado 28 infusiones mensuales, siendo la dosis de mantención actual $0,75 \mathrm{gr} / \mathrm{kg}$, repigmentándose casi completamente, con excepción de las manos, en las que este proceso ha sido más lento (Figuras $2 \mathrm{~A}$ y $2 \mathrm{~B})$.

\section{Discusión}

Los efectos terapéuticos de la IGIV en el vitiligo pudiesen ser resultado de la neutralización de autoanticuerpos contra múltiples antígenos, incluido tirosinasas, proteínas relacionadas con la tirosinasa 1 y 2, Pmel17, el factor de transcripción SOX, entre otros, los que han sido detectados en el suero de algunos pacientes con vitiligo ${ }^{6,7}$. Por su parte, está establecido que la IGIV regula los clones autorreactivos de linfocitos $\mathrm{B}^{8,9}$, controlando así la proliferación de éstos y modulando la producción de autoanticuerpos ${ }^{10}$. El bloqueo competitivo, es decir, la unión de la IgG proveniente de la IGIV en vez de los autoanticuerpos propios de los pacientes 

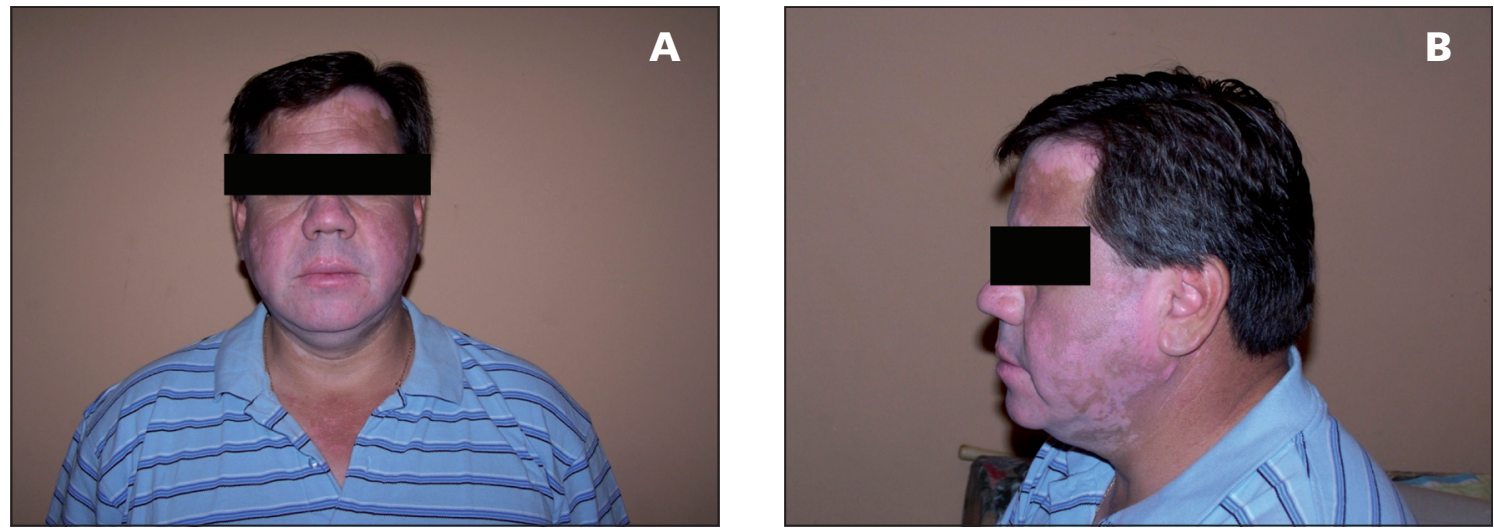

Figuras 1A y 1B. Fotografías del paciente, tanto de frente $(A)$ como de perfil (B), previo al inicio del tratamiento con IGIV.
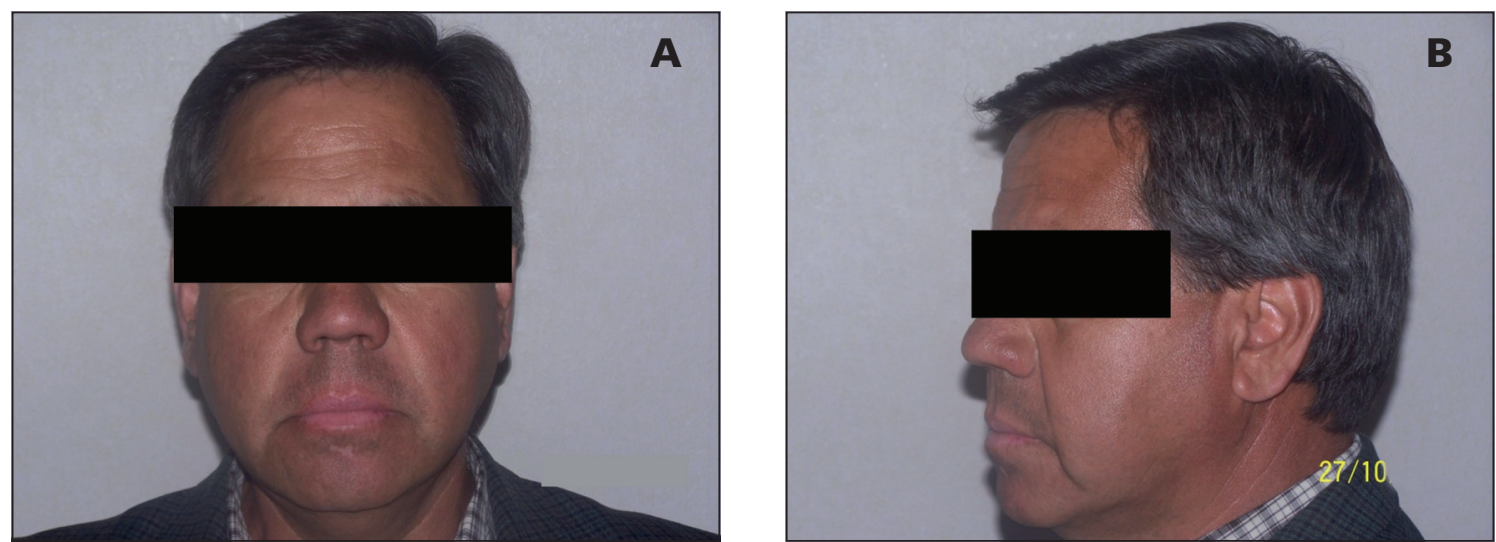

Figuras 2A y 2B. Fotografías del paciente, tanto de frente (A) como de perfil (B), posterior a 28 pulsos de IGIV.

con vitiligo a los receptores $\mathrm{Fc} \gamma \mathrm{R}$ en macrófagos, previene el clearance de los melanocitos sensibilizados (realizado por el sistema retículoendotelial), siendo considerado como el mecanismo clave de la IGIV en el control de las patologías mediadas por anticuerpos ${ }^{1,4,8,9,11-13}$.

En la piel con vitiligo destaca la presencia de LT CD8+ citotóxicos infiltrados en el área perilesional ${ }^{5,6} \mathrm{y}$ adyacentes a los melanocitos desaparecidos $^{15}$. También se han detectado LT CD8+ melanocitos-específicos circulantes, los cuales presentan en la superficie celular el antígeno CLA (cutaneous lymphocyte-asocciated), que permite el reclutamiento de estas células a la piel ${ }^{6}$. Otras células que infiltran, tanto el área con vitiligo como la perilesional, son los macrófagos, los que estarían implicados en la fagocitosis de los melanocitos apoptóticos $^{15,16}$.
A su vez, en los preparados de IGIV se han pesquisado anticuerpos anti-idiotipo que son capaces de unirse a la región variable del TCR (T-cell receptor), bloqueando su activación por autoantígenos; también están presentes anticuerpos naturales contra las molécula CD4, CD8 y contra una secuencia conservada de las moléculas de histocompatibilidad tipo I y II, que bloquean la proliferación de LT CD4 y CD8 respectivamente. La IGIV, a su vez, restaura el balance entre células Th1 y Th2 en enfermedades autoinmunes en las que la población de células autorreactivas Th1 o Th2 se encuentra expandida, al controlar su proliferación y modular su activación y la síntesis de citoquinas $^{12,13}$.

El vitiligo se asocia con niveles aumentados de interleucina-6 (inhibidor de la proliferación de melanocitos), interferón gamma (iniciador 
de la apoptosis) y factor de necrosis tumoral alfa (inhibidor de la proliferación de los melanocitos e iniciador de la apoptosis $)^{17,18}$. Por su parte, la expresión del factor estimulante de colonias de monocitos y granulocitos, de las células madres y el factor básico de crecimiento fibroblástico (cuya función es estimular a las células con pigmentos) es menor en la piel con vitiligo que en la piel perilesional, en la no lesionada así como en la de los controles sanos ${ }^{18}$. A su vez, se han encontrado una disminución en los niveles séricos del factor de crecimiento transformante beta en pacientes con vitiligo ${ }^{19}$, lo cual puede aumentar la respuesta de los LT CD8+ y disminuir la maduración de las células $\mathrm{T}$ reguladoras, causando deterioro en la inhibición de la inflamación en el vitiligo. La modulación en la producción de citoquinas por monocitos, macrófagos y LT CD4, así como de antagonistas de citoquinas, corresponde al mecanismo anti-inflamatorio más importante de la IGIV, permitiendo que el perfil clásico de citoquinas regrese a la normalidad ${ }^{4,11,13}$.

El beneficio de la IGIV observado en este paciente confirma el importante rol de la autoinmunidad en la inmunopatogenia del vitiligo. El uso de este producto en pacientes con esta patología puede abrir una alternativa terapéutica para pacientes que han recibido distintos tratamientos clásicos sin una respuesta adecuada. La IGIV es un fármaco seguro, con efectos adversos poco frecuentes y que pudiese ser utilizado en pacientes con alguna enfermedad autoinmune concomitante. Sin embargo, el gran inconveniente de esta terapia es su costo elevado y la necesidad de repetir las infusiones. En nuestro caso tenemos que destacar que a pesar de usar dosis bajas de IGIV, el vitiligo siempre fue mejorando, incluso al llegar a $0,5 \mathrm{~g} / \mathrm{kg}$, no así la neuropatía que presentó una reactivación. Podría plantearse entonces un tratamiento a demanda, con indicación de la dosis requerida e intervalos según los resultados clínicos.

\section{Referencias}

1. Lu T, Gao T, Wang A, Yin Y, Li Q, Li C. Vitiligo prevalence study in Shaanxi province China. Int J Dermatol 2007; 46: 47-51.

2. Rezaei N, Gavalas NG, Weetman AP, Keetman AP, Kemp $\mathrm{EH}$. Autoimmunity as an aetiological factor in vitiligo. J Eur Acad Dermatol Venereol 2007; 21: 865-76.
3. Gawkrodger DJ, Ormerod AD, Shaw L, Mauri-Sole I, Whitton ME, Whatts MJ, et al. Guideline for the diagnosis and management of vitiligo. Br J Dermatol 2008; 159: 1051-76.

4. Negi VS, Elluru S, Sibéril S, Graff-Dubois S, Mouthon $\mathrm{L}$, Kazatchkine MD, et al. Intravenous Immunoglobulin: An Update on the Clinical Use and Mechanism of Action. J Clin Immunol 2007; 27: 233-45.

5. Nimmerjahn F, Ravetch V. Anti-Inflamatory Actions of Intravenous Immunoglobulin. Annu Rev Immunol 2008; 26: 513-33.

6. Ongenae K, Van Geel N, Naeyaert JM. Evidence for an autoimmune pathogenesis of vitiligo. Pigment Cell Res 2003; 16: 90-100.

7. Kemp EH, Waterman EA, Hawes BE, O’Neill K, Gottumukkala RV, Gawkrodger DJ, et al. The melaninconcentrating hormone receptor 1 , a novel target of autoantibody responses in vitiligo. J Clin Invest 2002; 109: 923-30.

8. Simon HU, Spath PJ. IVIG - mechanism of action. Allergy 2003; 58: 543-52.

9. Lemieux R, Bazin R, Néron S. Therapeutic intravenous immunoglobilins. Molecular Immunology 2005; 42: 839-48.

10. Kazatchkine M, Kaveri S. Immunomodulation of Autoimmune and Inflammatory Diseases with Intravenous Immune Globulin. N Engl J Med 2001; 345: 747-53.

11. Prins Ch, Gelfand E, French L. Intravenous Immunoglobulin: Properties, Mode of Action and Practical use in Dermatology. Acta Derm Venereol 2007; 87: 206-18.

12. Kazatchkine M, Kaveri S. Immunomodulation of Autoimmune and Inflammatory Diseases with Intravenous Immune Globulin. N Engl J Med 2001; 345: 747-53.

13. Siberil S, Elluru S, Graff-Dubois S, Negi VS, Delignat $S$, Mouthon L, et al. Intravenous Immunoglobulins in Autoimmune and Inflammatory disease: a mechanistic perspective. Ann N Y Acad Sci 2007; 1110: 497-506.

14. Le Poole IC, van den Wijngaard RM, Westerhof W, Das PK. Presence of T cells and macrophages in inflammatory vitiligo skin parallels melanocyte disappearance. Am J Pathol 1996; 148: 1219-28.

15. Van den Wijngaard R, Wankowicz-Kalinska A, Le Poole C, Tigges B, Westerhof W, Das P. Local immune response in skin of generalised vitiligo patients. Destruction of melanocytes is associated with the prominent presence of CLA+ T cells at the perilesional site. Lab Invest 2000; 80: 1299-309.

16. LePoole IC, van den Wijngaard RM, Westerhof W, Das PK. Presence of T cells and macrophages in inflammatory vitiligo skin parallels melanocyte disappearance. Am J Pathol 1996; 148: 1219-28. 
17. Huang CL, Nordlund JJ, Boissy R. Vitiligo: a manifestation of apoptosis?. Am J Clin Dermatol 2002; 3: 301-8.

18. Grimes PE, Morris R, Avaniss-Aghajani E, Soriano T, Meraz M, Metzger A. Topical tacrolimus therapy for vitiligo: therapeutic responses and skin messenger RNA expression of proinflammatory cytokines. J Am Acad Dermatol 2004; 51: 52-61.

19. Basak PY, Adiloglu AK, Ceyhan AM, Tas T, Akkaya VB. The role of helper and regulatory $\mathrm{T}$ cells in the pathogenesis of vitiligo. J Am Acad Dermatol 2009; 60: 256-60. 